\title{
Freeway Traffic Density and On-Ramp Queue Control via ILC Approach
}

\author{
Ronghu Chi, ${ }^{1}$ Mengze Li, ${ }^{2}$ Zhongsheng Hou, ${ }^{3}$ Xiangpeng Liu, ${ }^{4}$ and Zhaoxu $\mathrm{Yu}^{2}$ \\ ${ }^{1}$ School of Automation \& Electronics Engineering, Qingdao University of Science \& Technology, Qingdao 266042, China \\ ${ }^{2}$ School of Information Science and Engineering, East China University of Science \& Technology, Shanghai 201424, China \\ ${ }^{3}$ School of Electronics \& Information Engineering, Beijing Jiaotong University, Beijing 100044, China \\ ${ }^{4}$ School of Polymer Science and Engineering, Qingdao University of Science \& Technology, Qingdao 266042, China
}

Correspondence should be addressed to Ronghu Chi; ronghu_chi@hotmail.com

Received 18 January 2014; Accepted 21 March 2014; Published 14 April 2014

Academic Editor: Youqing Wang

Copyright (c) 2014 Ronghu Chi et al. This is an open access article distributed under the Creative Commons Attribution License, which permits unrestricted use, distribution, and reproduction in any medium, provided the original work is properly cited.

\begin{abstract}
A new queue length information fused iterative learning control approach (QLIF-ILC) is presented for freeway traffic ramp metering to achieve a better performance by utilizing the error information of the on-ramp queue length. The QLIF-ILC consists of two parts, where the iterative feedforward part updates the control input signal by learning from the past control data in previous trials, and the current feedback part utilizes the tracking error of the current learning iteration to stabilize the controlled plant. These two parts are combined in a complementary manner to enhance the robustness of the proposed QLIF-ILC. A systematic approach is developed to analyze the convergence and robustness of the proposed learning scheme. The simulation results are further given to demonstrate the effectiveness of the proposed QLIF-ILC.
\end{abstract}

\section{Introduction}

Freeway traffic control [1-3] is an important area in the field of traffic engineering and intelligent transportation systems. Frequent congestion on freeway during rush hours deteriorates traffic conditions. The most common reasons causing freeway congestion include traffic demand being greater than capacity, as well as traffic accidents, road works, and weather [3]. For better utilization of freeway capacity, ramp metering $[2,4-7]$ is a commonly implemented strategy. The purpose of ramp metering is to regulate the amount of traffic entering a given freeway at its entry ramps to maximize throughput by maintaining a desired (or optimal) occupancy on the downstream mainline freeway.

Recently, various model-based control methods were developed to solve the problem, for instance, numerical methods $[4,5]$, function approximation based on neural network $[6,8]$, linearization method [2], one-step ahead prediction and multiple prediction adaptive control [7], and so forth. A limitation of model-based control is its sensitivity to modeling accuracy. Note that the scale of freeway traffic system expands with its dynamics becoming more complex nowadays. Consequently, an accurate freeway traffic model is hardly available in practice and it is highly nonlinear. Thus, model-based control methods may not be able to produce satisfactory performance. It is desirable to develop a new control method that is less dependent on the model accuracy.

It is noted that traffic flow patterns are in general repeated every day. For instance, the traffic flow will start from a very low level at midnight and increase gradually up to the first peak of morning rush hour, which is often from 7 to 9 a.m., and the second one, which is from 5 to 7 p.m. [9]. Congestion typically starts at the same locations every day. Based on this observation, the iterative learning control (ILC) based ramp metering strategy has been proposed for the freeway density control [9-11]. Iterative learning control was first proposed in [12] for the control of a system that repeats the same task in a finite interval and has been extensively studied and achieved significant progress in both theory and applications [13-17]. ILC has a very simple structure and requires very little system knowledge, which is a very desirable feature in traffic control since the traffic model and the exogenous factors may not be well known in practice. 
In [9], only the pure ILC based ramp metering and speed control were discussed. In [10], the learning mechanism combined with an error feedback in a complementary manner was studied and the simulation results have shown its superiority to the pure ILC scheme. Recently, the ILC based ramp metering and the modified modularized ramp metering based on ILC and ALINEA in the presence of input constraints were also analyzed to enhance the robustness of the proposed methods [11].

It is worth pointing out that the control input signals in [9-11] are updated based on the error information of the freeway traffic density only. Actually, the queue length of on-ramp is a significant factor [18]. Whenever the on-ramp demand exceeds the desired metering rate, a queue will form. However, the storage capacity of an on-ramp is often very limited. Therefore, if without proper control, the vehicle queue length will quickly exceed this capacity, causing the vehicles to spill over into the surface streets and interfere with street traffic.

Note that the current work is to consider the queuing demands as a constraint on the ramp metering [9-11]; that is, the on-ramp volumes cannot exceed the current demands plus the existing waiting queues at on-ramps. However, it is a queue-override scheme in nature [18]. If the queue detector occupancy is above the threshold, the metering rate is increased by a certain level. When the queue detector occupancy falls below the threshold, the metering rate is reset to the value determined by the controller. It has been noted $[19,20]$ that this queue-override scheme leads to oscillatory behavior and underutilization of on-ramp storage capacities.

In this paper, we present a new queue length information fusion based iterative learning control approach (QLIFILC) for freeway traffic responsive ramp-metering by using the repeated traffic patterns. The proposed control scheme consists of a feedforward learning part, used to learn from the past control data in the previous trial to meet the performance requirement, and a feedback stabilizing parts, designed to restrain the state error and input disturbance such that the closed-loop output tracking error is within a reasonable bound. A most distinct feature of the proposed QLIF-ILC is that the error information of the on-ramp queue length is used to prevent excessively long queues from interfering with surface street traffic and achieve a better performance. Both the rigorous analysis and the simulation results are given to demonstrate the efficiency and applicability of the proposed QLIF-ILC.

The rest of this paper is organized as follows. Section 2 introduces the traffic flow model and gives the problem formulation. The iterative learning controller based on the queue length information is designed and analyzed in Section 3. Section 4 provides some simulation results to illustrate the applicability and effectiveness of the proposed approach. Finally, some conclusions are given in Section 5.

\section{Traffic Flow Model and Problem Formulation}

The macroscopic traffic flow model [21] of a section is shown in Figure 1, where $f(k)$ is the traffic flow entering the section

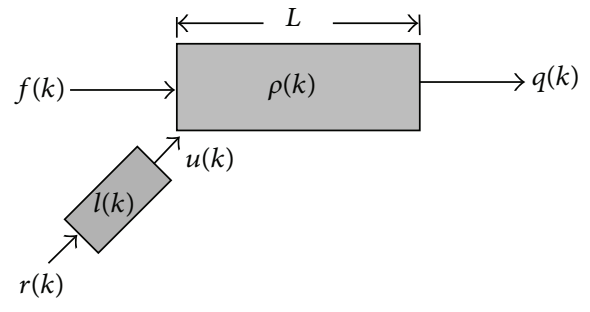

Figure 1: Freeway ramp system.

(veh/hour), $q(k)$ is the traffic flow leaving the section (veh/ hour), $\rho(k)$ is the traffic flow density at time $k$ (veh/lane $/ \mathrm{km}$ ), $u(k)$ is the flow rate entering the mainline from the ramp (veh/hour), $l(k)$ is the queue length in the ramp, $r(k)$ is the inflow entering the ramp (veh/hour), $L$ is the length of the section, and $k$ is the time instant.

Let the total number of vehicles in the section at time instant $k$ be given by $N(k)$. At time $(k+1)$, the number of vehicles is given by $N(k+1)$. Accordingly, the traffic density $\rho(k)$ is changed to be $\rho(k+1)$ and thus we have

$$
N(k+1)-N(k)=(\rho(k+1)-\rho(k)) \cdot L \cdot m,
$$

where $m$ is the number of lanes of the freeway mainline.

In addition, the change in the number of vehicles in the section can be expressed as

$$
N(k+1)-N(k)=(-q(k)+u(k)+f(k)) \cdot T,
$$

where $T$ is the sampling time.

Apparently, it is easy to obtain from (1) and (2) that

$$
(\rho(k+1)-\rho(k)) \cdot L \cdot m=(-q(k)+u(k)+f(k)) \cdot T,
$$

or

$$
\rho(k+1)=\rho(k)+\frac{T}{m L}(-q(k)+u(k)+f(k)) .
$$

According to Greenshield's model [22], one has

$$
v(k)=v_{f}\left(1-\frac{\rho(k)}{\rho_{\max }}\right),
$$

where $\rho_{\max }$ is the maximum possible density per lane and $v_{f}$ is the free speed. Thus, the relationship among traffic flow, density, and velocity can be expressed as

$$
q(k)=m \rho(k) v(k)=m \rho(k) v_{f}\left(1-\frac{\rho(k)}{\rho_{\max }}\right) .
$$

Substituting (6) into (4) yields

$$
\begin{aligned}
\rho(k+1)= & \left(v_{f} \frac{T}{L} \frac{\rho^{2}(k)}{\rho_{\max }}-v_{f} \frac{T}{L} \rho(k)+\rho(k)\right)+\frac{T}{m L} u(k) \\
& +\frac{T}{m L} f(k) .
\end{aligned}
$$


According to the conservation law, the number of vehicles that stay in the on-ramp at time $k$ is equal to the difference between the inflow volume and outflow volume; that is,

$$
l(k+1)=l(k)+T(r(k)-u(k)) .
$$

From (7) and (8), we have the macroscopic traffic model as follows:

$$
\begin{aligned}
& {\left[\begin{array}{c}
\rho(k+1) \\
l(k+1)
\end{array}\right]=\left[\begin{array}{c}
v_{f} \frac{T}{L} \frac{\rho^{2}(k)}{\rho_{\max }}-v_{f} \frac{T}{L} \rho(k)+\rho(k) \\
l(k)
\end{array}\right]} \\
& +\left[\begin{array}{c}
\frac{T}{m L} \\
-T
\end{array}\right] u(k)+\left[\begin{array}{cc}
\frac{T}{m L} & 0 \\
0 & T
\end{array}\right]\left[\begin{array}{c}
f(k) \\
r(k)
\end{array}\right] \text {. }
\end{aligned}
$$

Let $\mathbf{x}(k)=\left[\begin{array}{l}\rho(k) \\ l(k)\end{array}\right], \mathbf{b}=\left[\begin{array}{c}T / m L \\ -T\end{array}\right], \mathbf{D}=\left[\begin{array}{cc}T / m L & 0 \\ 0 & T\end{array}\right], \mathbf{s}(k)=$ $\left[\begin{array}{l}f(k) \\ r(k)\end{array}\right]$, and $\mathbf{C}=\left[\begin{array}{ll}1 & 0 \\ 0 & 1\end{array}\right]$; we can rewrite $(9)$ as

$$
\begin{gathered}
\mathbf{x}(k+1)=\mathbf{g}(k, \mathbf{x}(k))+\mathbf{b} u(k)+\operatorname{Ds}(k), \\
\mathbf{y}(k)=\mathbf{C} \mathbf{x}(k),
\end{gathered}
$$

where $\mathbf{g}\left(k, \mathbf{x}_{n}(k)\right)$ is a corresponding vector-valued function and $\mathbf{y}(k)$ is the system output.

To show the repeatable property and the strong uncertainties of the freeway traffic system, we denote the above macroscopic traffic flow model as follows:

$$
\begin{gathered}
\mathbf{x}_{n}(k+1)=\mathbf{g}\left(k, \mathbf{x}_{n}(k)\right)+\mathbf{b} u_{n}(k)+\operatorname{Ds}(k)+\psi_{n}(k), \\
\mathbf{y}_{n}(k)=\mathbf{C} \mathbf{x}_{n}(k)+\boldsymbol{\eta}_{n}(k),
\end{gathered}
$$

where $\boldsymbol{\psi}_{n}(k)$ and $\boldsymbol{\eta}_{n}(k)$ are added as the bounded state disturbances and output noises, respectively, $n$ denotes the iteration number, $k \in\{0,1, \ldots, K\}$ belongs to a finite time interval, and $\mathbf{s}(k)$ is regarded as an unknown repeatable disturbance in the following controller design and analysis.

The control objective of this work is to find a proper control input $u_{n}(k)$ driving the traffic flow density to track the desired traffic density $\rho_{d}$ and the desired queue length of the on-ramp, $l_{d}$.

Remark 1. In this paper, the control objective considered is only for some idea cases, where the traffic demand is not too heavy and one can manipulate the flow rate entering the mainline from the on-ramp to achieve the desired traffic density and to arrive at the desired queue length meanwhile.

Remark 2. In fact, there are more practical cases where the traffic demands of the mainline and on-ramp are heavy but not causing congestion. For such cases, it is better to consider a balance between the traffic density of the mainline and the queue length of the on-ramp by setting their proper desired values. Then, the control objective is to make the traffic density and the queue length track the desired values, respectively, as much as possible and the final tracking errors may not be zeros but some minimum values.

\section{Controller Design and Convergence Analysis}

3.1. Controller Design. Design a PD-type ILC law based on the queue length information fusion (QLIF-ILC) as follows:

$$
u_{n+1}(k)=u_{n}(k)+\Gamma_{1}\left[\mathbf{e}_{n}(k+1)-\mathbf{e}_{n}(k)\right]+\Gamma_{2} \mathbf{e}_{n+1}(k),
$$

where $\boldsymbol{\Gamma}_{1}$ and $\boldsymbol{\Gamma}_{2}$ are learning gain vectors and $\mathbf{e}_{n}(k+1)=$ $\mathbf{y}_{d}(k+1)-\mathbf{y}_{n}(k+1)$ with $\mathbf{y}_{d}(k)$ being the desired output at time $k$.

Remark 3. Expand control law (12) as

$$
\begin{aligned}
u_{n+1}(k)= & u_{n}(k)+\Gamma_{11}\left(e_{n}^{\rho}(k+1)-e_{n}^{\rho}(k)\right) \\
& +\Gamma_{12}\left(e_{n}^{l}(k+1)-e_{n}^{l}(k)\right)+\Gamma_{21} e_{n+1}^{\rho}(k) \\
& +\Gamma_{22} e_{n+1}^{l}(k),
\end{aligned}
$$

where $e_{n}^{\rho}(k)=\rho_{d}(k)-\rho_{n}(k)$ is the tracking error of the traffic density and $e_{n}^{l}(k)=l_{d}(k)-l_{n}(k)$ is the tracking error of the queue length of the on-ramp. Hence, the weighted sum of the tracking errors of the traffic density and the queue length is utilized in the QLIF-ILC to achieve a better performance.

Remark 4. The proposed ILC approach consists of a feedforward learning part $\boldsymbol{\Gamma}_{1}\left[\mathbf{e}_{n}(k+1)-\mathbf{e}_{n}(k)\right]$ and a feedback stabilizing part $\boldsymbol{\Gamma}_{2} \mathbf{e}_{n+1}(k)$. The feedforward learning part is designed to meet the performance requirement. And the feedback stabilizing part is designed to accommodate the uncertainties and disturbances.

3.2. Convergence Analysis. Before the convergence analysis, some assumptions should be given as follows for the rigorous analysis.

Assumption 5. There exists a control input $u_{d}(k)$ such that the system output can be driven to track the desired trajectory $\mathbf{y}_{d}(k)$ for the system (11) over the finite time interval $\{0,1, \ldots, K\}$.

Assumption 6. The reinitialization condition is satisfied throughout the repeated iterations; that is,

$$
\mathbf{x}_{n}(0)=\mathbf{x}_{d}(0), \quad \mathbf{y}_{n}(0)=\mathbf{y}_{d}(0), \quad \forall n,
$$

where $\mathbf{x}_{d}(0)$ and $\mathbf{y}_{d}(0)$ are the initial values of the desired states.

Assumption 7. The function $\mathbf{g}(k, \mathbf{x}(k))$ is Lipschitz continuous; that is,

$$
\left\|\mathbf{g}\left(k, \mathbf{x}_{1}(k)\right)-\mathbf{g}\left(k, \mathbf{x}_{2}(k)\right)\right\| \leq M\left\|\mathbf{x}_{1}(k)-\mathbf{x}_{2}(k)\right\|,
$$

where $M>0$ is the Lipschitz constant. 
Assumption 8. All of $\mathbf{g}\left(k, \mathbf{x}_{n}(k)\right), \mathbf{b}, \boldsymbol{\psi}_{n}(k)$, and $\boldsymbol{\eta}_{n}(k)$ are bounded; that is,

$$
\begin{aligned}
& \sup _{n} \max _{k \in\{0,1, \ldots, K\}}\left\{\left\|\mathbf{g}\left(k, \mathbf{x}_{n}(k)\right)\right\|\right\} \leq b_{\mathbf{g}}, \quad\|\mathbf{b}\| \leq b_{\mathbf{b}}, \\
& \sup _{n} \max _{k \in\{0,1, \ldots, K\}}\left\{\left\|\psi_{n}(k)\right\|\right\} \leq b_{\psi}, \\
& \sup _{n} \max _{k \in\{0,1, \ldots, K\}}\left\{\left\|\boldsymbol{\eta}_{n}(k)\right\|\right\} \leq b_{\boldsymbol{\eta}},
\end{aligned}
$$

where $b_{\mathbf{g}}, b_{\mathbf{b}}, b_{\psi}$, and $b_{\eta}$ are some positive constants.

Remark 9. Assumption 5 is very common in the control theory field. Assumption 6 is the standard condition for ILC analysis using contraction mapping approach. Although there exists a quadratic term in $\mathbf{g}(k, \mathbf{x}(k))$, it is noted that $\rho(k)$ is always less than $\rho_{\text {jam }}$. So, Assumption 7 is automatically satisfied for the freeway traffic system. Note that, in Assumption 8, we just need the existence of the positive constants instead of their exact values.

The validity of the above presented optimal terminal ILC is verified by the following theorem.

Theorem 10. Under Assumptions 5-8, choosing the learning gain matrix $\boldsymbol{\Gamma}_{1}$ such that $\left\|\mathbf{I}-\boldsymbol{\Gamma}_{1} \mathbf{C b}\right\| \leq \lambda<1$ and applying the proposed QLIF-ILC law (12) to the freeway traffic system (11), one can guarantee that $\lim _{n \rightarrow \infty}\left\|\mathbf{e}_{n}\right\|_{\alpha} \leq \sigma$ for some positive constant $\sigma$ depending on $b_{\mathrm{g}}, b_{\mathbf{b}}, b_{\psi}, b_{\eta}, \Gamma_{1}, \Gamma_{2}, \alpha$, and $M$. If $\psi_{n}(k)=0$ and $\boldsymbol{\eta}_{n}(k)=0$, one can guarantee that the tracking error converges to zero as iteration number approaches to infinity; that is, $\lim _{n \rightarrow \infty} \mathbf{e}_{n}(k)=0, k \in\{0,1, \ldots, K\}$.

Proof. Let $\Delta \mathbf{x}_{n}(k)=\mathbf{x}_{d}(k)-\mathbf{x}_{n}(k), \Delta u_{n}(k)=u_{d}(k)-u_{n}(k)$; then,

$$
\begin{aligned}
\Delta \mathbf{x}_{n}(k)= & \mathbf{x}_{d}(k)-\mathbf{x}_{n}(k) \\
= & {\left[\mathbf{g}\left(k, \mathbf{x}_{d}(k-1)\right)+\mathbf{b} u_{d}(k-1)+\mathbf{D s}(k-1)\right] } \\
& -\left[\mathbf{g}\left(k, \mathbf{x}_{n}(k-1)\right)+\mathbf{b} u_{n}(k-1)\right. \\
& \left.\quad+\mathbf{D s}(k-1)+\psi_{n}(k-1)\right] \\
= & \mathbf{g}\left(k, \mathbf{x}_{d}(k-1)\right)-\mathbf{g}\left(k, \mathbf{x}_{n}(k-1)\right) \\
& +\mathbf{b} \Delta u_{n}(k-1)-\psi_{n}(k-1) .
\end{aligned}
$$

According to Assumption 7, taking norm on both sides of (17) yields

$$
\begin{aligned}
\left\|\Delta \mathbf{x}_{n}(k)\right\| \leq & M\left\|\mathbf{x}_{d}(k-1)-\mathbf{x}_{n}(k-1)\right\| \\
& +b_{\mathbf{b}}\left\|\Delta u_{n}(k-1)\right\|+b_{\psi} \\
= & M\left\|\Delta \mathbf{x}_{n}(k-1)\right\|+b_{\mathbf{b}}\left\|\Delta u_{n}(k-1)\right\|+b_{\psi} \\
= & M^{k}\left\|\Delta \mathbf{x}_{n}(0)\right\|+\sum_{j=0}^{k-1} M^{k-1-j}\left(b_{\mathbf{b}}\left\|\Delta u_{n}(j)\right\|+b_{\psi}\right) .
\end{aligned}
$$

In terms of Assumption 6, (18) can be rewritten as

$$
\left\|\Delta \mathbf{x}_{n}(k)\right\| \leq \sum_{j=0}^{k-1} M^{k-1-j}\left(b_{\mathbf{b}}\left\|\Delta u_{n}(j)\right\|+b_{\psi}\right) .
$$

From (11), the error dynamics is given as

$$
\begin{aligned}
\mathbf{e}_{n}(k)=\mathbf{y}_{d}(k)-\mathbf{y}_{n}(k) & =\mathbf{C} \mathbf{x}_{d}(k)-\mathbf{C} \mathbf{x}_{n}(k)-\boldsymbol{\eta}_{n}(k) \\
& =\mathbf{C} \Delta \mathbf{x}_{n}(k)-\boldsymbol{\eta}_{n}(k) .
\end{aligned}
$$

It is easy to obtain from (11), (12), and (20) that

$$
\begin{aligned}
& \Delta u_{n+1}(k) \\
& =u_{d}(k)-u_{n+1}(k) \\
& =u_{d}(k)-\left(u_{n}(k)+\Gamma_{1}\left[\mathbf{e}_{n}(k+1)-\mathbf{e}_{n}(k)\right]+\Gamma_{2} \mathbf{e}_{n+1}(k)\right) \\
& =\Delta u_{n}(k)-\Gamma_{1}\left(\mathbf{y}_{d}(k+1)-\mathbf{y}_{d}(k)-\mathbf{y}_{n}(k+1)+\mathbf{y}_{n}(k)\right) \\
& -\Gamma_{2} \mathbf{e}_{n+1}(k) \\
& =\Delta u_{n}(k)-\Gamma_{1}\left(\mathbf{C x}_{d}(k+1)-\mathbf{C x}_{n}(k+1)-\boldsymbol{\eta}_{n}(k+1)\right. \\
& \left.-\mathbf{C x}_{d}(k)+\mathbf{C} \mathbf{x}_{n}(k)+\boldsymbol{\eta}_{n}(k)\right)-\boldsymbol{\Gamma}_{2} \mathbf{e}_{n+1}(k) \\
& =\Delta u_{n}(k)-\Gamma_{1} \mathbf{C}\left(\left(\mathbf{g}\left(k, \mathbf{x}_{d}(k)\right)+\mathbf{b} u_{d}(k)+\operatorname{Ds}(k)\right)\right. \\
& \left.-\left(\mathbf{g}\left(k, \mathbf{x}_{n}(k)\right)+\mathbf{b} u_{n}(k)+\mathbf{D s}(k)\right)\right) \\
& +\Gamma_{1} \mathbf{C} \psi_{n}(k)+\Gamma_{1}\left(\boldsymbol{\eta}_{n}(k+1)-\boldsymbol{\eta}_{n}(k)\right) \\
& +\Gamma_{1} \mathbf{C}\left(\mathbf{x}_{d}(k)-\mathbf{x}_{n}(k)\right)-\boldsymbol{\Gamma}_{2} \mathbf{e}_{n+1}(k) \\
& =\left(\mathbf{I}-\boldsymbol{\Gamma}_{1} \mathbf{C b}\right) \Delta u_{n}(k)-\boldsymbol{\Gamma}_{1} \mathbf{C}\left(\mathbf{g}\left(k, \mathbf{x}_{d}(k)\right)-\mathbf{g}\left(k, \mathbf{x}_{n}(k)\right)\right) \\
& +\Gamma_{1}\left(\mathbf{C} \psi_{n}(k)+\boldsymbol{\eta}_{n}(k+1)-\boldsymbol{\eta}_{n}(k)\right) \\
& +\boldsymbol{\Gamma}_{1} \mathbf{C}\left(\mathbf{x}_{d}(k)-\mathbf{x}_{n}(k)\right)-\boldsymbol{\Gamma}_{2} \mathbf{e}_{n+1}(k) \text {. }
\end{aligned}
$$

Select $\boldsymbol{\Gamma}_{1}$ such that $\left\|\mathbf{I}-\boldsymbol{\Gamma}_{1} \mathbf{C b}\right\| \leq \lambda<1 ; \lambda$ is a positive constant. Let $\mathbf{b}_{\mathbf{\Gamma}_{M}}=\left\|\boldsymbol{\Gamma}_{1} \mathbf{C M}+\boldsymbol{\Gamma}_{1} \mathbf{C}\right\|, b_{\boldsymbol{\Gamma}_{1}}=\left\|\boldsymbol{\Gamma}_{1} \mathbf{C}\right\|, b_{\boldsymbol{\Gamma}_{2}}=\left\|\boldsymbol{\Gamma}_{2} \mathbf{C}\right\|$, $\mathbf{b}_{1}=\left\|\boldsymbol{\Gamma}_{1}\right\|, b_{2}=\left\|\boldsymbol{\Gamma}_{2}\right\|$. In terms of Assumption 8, we know that $b_{\mathbf{b}}, b_{\psi}$, and $b_{\eta}$ are bounded. Taking norm on both sides of (21) and using (19) and (20), we have

$$
\begin{aligned}
& \left\|\Delta u_{n+1}(k)\right\| \\
& \leq\left\|\mathbf{I}-\boldsymbol{\Gamma}_{1} \mathbf{C b}\right\|\left\|\Delta u_{n}(k)\right\| \\
& \quad+\left\|\boldsymbol{\Gamma}_{1} \mathbf{C M}+\boldsymbol{\Gamma}_{1} \mathbf{C}\right\|\left\|\Delta \mathbf{x}_{n}(k)\right\| \\
& \quad+\left\|\boldsymbol{\Gamma}_{1} \mathbf{C}\right\| b_{\psi}+2\left\|\boldsymbol{\Gamma}_{1}\right\| b_{\boldsymbol{\eta}} \\
& \quad+\left\|\boldsymbol{\Gamma}_{2} \mathbf{C}\right\|\left\|\Delta \mathbf{x}_{n+1}(k)\right\|+\left\|\boldsymbol{\Gamma}_{2}\right\| b_{\eta}
\end{aligned}
$$




$$
\begin{aligned}
\leq & \left\|\mathbf{I}-\Gamma_{1} \mathbf{C b}\right\|\left\|\Delta u_{n}(k)\right\|+b_{\Gamma_{M}}\left\|\Delta \mathbf{x}_{n}(k)\right\| \\
& +b_{\Gamma_{1}} b_{\psi}+2 b_{1} b_{\eta}+b_{\Gamma_{2}}\left\|\Delta \mathbf{x}_{n+1}(k)\right\|+b_{2} b_{\eta} \\
\leq & \lambda\left\|\Delta u_{n}(k)\right\|+b_{\Gamma_{M}}\left[\sum_{j=0}^{k-1} M^{k-1-j}\left(b_{\mathbf{b}}\left\|\Delta u_{n}(j)\right\|+b_{\psi}\right)\right] \\
& +b_{\Gamma_{2}}\left[\sum_{j=0}^{k-1} M^{k-1-j}\left(b_{\mathbf{b}}\left\|\Delta u_{n+1}(j)\right\|+b_{\psi}\right)\right] \\
& +b_{\Gamma_{1}} b_{\psi}+\left(2 b_{1}+b_{2}\right) b_{\eta} \\
\leq & \lambda\left\|\Delta u_{n}(k)\right\|+b_{\Gamma_{M}} \sum_{j=0}^{k-1} M^{k-1-j}\left(b_{\mathbf{b}}\left\|\Delta u_{n}(j)\right\|+b_{\psi}\right) \\
& +b_{\Gamma_{2}} \sum_{j=0}^{k-1} M^{k-1-j}\left(b_{\mathbf{b}}\left\|\Delta u_{n+1}(j)\right\|+b_{\psi}\right) \\
& +b_{\Gamma_{1}} b_{\psi}+\left(2 b_{1}+b_{2}\right) b_{\eta} .
\end{aligned}
$$

Define $\alpha$-norm as

$$
\left\|\Delta u_{n+1}\right\|_{\alpha}=\left\|\Delta u_{n+1}(k)\right\|\left(\frac{1}{\alpha}\right)^{k} .
$$

Multiplying $(1 / \alpha)^{k}$ for both sides of (22), one obtains

$$
\begin{aligned}
& \left\|\Delta u_{n+1}(k)\right\|\left(\frac{1}{\alpha}\right)^{k} \\
& \leq \lambda\left\|\Delta u_{n}(k)\right\|\left(\frac{1}{\alpha}\right)^{k} \\
& +\left(\frac{b_{\Gamma_{M}}}{\alpha}\right) \sum_{j=0}^{k-1}\left(\frac{M}{\alpha}\right)^{k-1-j}\left[b_{\mathbf{b}}\left\|\Delta u_{n}(j)\right\|\left(\frac{1}{\alpha}\right)^{j}+\left(\frac{1}{\alpha}\right)^{j} b_{\psi}\right] \\
& +\left(\frac{b_{\Gamma_{2}}}{\alpha}\right)^{k-1} \sum_{j=0}^{k}\left(\frac{M}{\alpha}\right)^{k-1-j}\left[b_{\mathbf{b}}\left\|\Delta u_{n+1}(j)\right\|\left(\frac{1}{\alpha}\right)^{j}+\left(\frac{1}{\alpha}\right)^{j} b_{\psi}\right] \\
& \quad+\left(\frac{1}{\alpha}\right)^{k}\left[b_{\Gamma_{1}} b_{\psi}+\left(2 b_{1}+b_{2}\right) b_{\eta}\right] .
\end{aligned}
$$

Selecting $\alpha>\max \{1, M\}$, one can obtain from (24) that $\left\|\Delta u_{n+1}\right\|_{\alpha}$

$$
\begin{aligned}
\leq & \lambda\left\|\Delta u_{n}\right\|_{\alpha}+\left(b_{\mathbf{b}}\left\|\Delta u_{n}\right\|_{\alpha}+b_{\psi}\right)\left(\frac{b_{\Gamma_{M}}}{\alpha}\right) \sum_{j=0}^{k-1}\left(\frac{M}{\alpha}\right)^{k-1-j} \\
& +\left(b_{\mathbf{b}}\left\|\Delta u_{n+1}\right\|_{\alpha}+b_{\psi}\right)\left(\frac{b_{\Gamma_{2}}}{\alpha}\right) \sum_{j=0}^{k-1}\left(\frac{M}{\alpha}\right)^{k-1-j} \\
& +b_{\Gamma_{1}} b_{\psi}+\left(2 b_{1}+b_{2}\right) b_{\eta}
\end{aligned}
$$

$$
\begin{aligned}
& \leq\left(\lambda+b_{\mathbf{b}} b_{\Gamma_{M}} \frac{1-(M / \alpha)^{K}}{\alpha-M}\right)\left\|\Delta u_{n}\right\|_{\alpha} \\
& +b_{\mathbf{b}} b_{\Gamma_{2}} \frac{1-(M / \alpha)^{K}}{\alpha-M}\left\|\Delta u_{n+1}\right\|_{\alpha} \\
& \quad+b_{\psi}\left\{\frac{\left(b_{\Gamma_{M}}+b_{\Gamma_{2}}\right)\left[1-(M / \alpha)^{K}\right]}{\alpha-M}+b_{\Gamma_{1}}\right\}+\left(2 b_{1}+b_{2}\right) b_{\eta} .
\end{aligned}
$$

Rearranging (25) yields

$$
\begin{aligned}
\left(1-b_{\mathbf{b}} b_{\Gamma_{2}} \frac{1-(M / \alpha)^{K}}{\alpha-M}\right)\left\|\Delta u_{n+1}\right\|_{\alpha} \\
\leq\left(\lambda+b_{\mathbf{b}} b_{\Gamma_{M}} \frac{1-(M / \alpha)^{K}}{\alpha-M}\right)\left\|\Delta u_{n}\right\|_{\alpha} \\
\quad+b_{\psi}\left\{\frac{\left(b_{\Gamma_{M}}+b_{\Gamma_{2}}\right)\left[1-(M / \alpha)^{K}\right]}{\alpha-M}+b_{\Gamma_{1}}\right\} \\
+\left(2 b_{1}+b_{2}\right) b_{\eta} .
\end{aligned}
$$

Let $\alpha>\max \left\{1, M, M+b_{\mathbf{b}} b_{\boldsymbol{\Gamma}_{2}}\right\}$; then (26) becomes

$$
\left\|\Delta u_{n+1}\right\|_{\alpha} \leq \hat{\lambda}\left\|\Delta u_{n}\right\|_{\alpha}+\varepsilon
$$

where

$$
\begin{gathered}
\hat{\lambda}=\frac{\lambda+b_{\mathbf{b}} b_{\Gamma_{M}}\left(\left(1-(M / \alpha)^{K}\right) /(\alpha-M)\right)}{1-b_{\mathbf{b}} b_{\Gamma_{2}}\left(\left(1-(M / \alpha)^{K}\right) /(\alpha-M)\right)}>0, \\
\varepsilon=\left(\left(b_{\psi}\left\{\frac{\left(b_{\Gamma_{M}}+b_{\Gamma_{2}}\right)\left[1-(M / \alpha)^{K}\right]}{\alpha-M}+b_{\Gamma_{1}}\right\}\right.\right. \\
\left.+\left(2 b_{1}+b_{2}\right) b_{\eta} b_{\psi}\right) \\
\left.\quad \times\left(1-b_{\mathbf{b}} b_{\Gamma_{2}} \frac{1-(M / \alpha)^{K}}{\alpha-M}\right)^{-1}\right)>0 .
\end{gathered}
$$

It is obvious from (27) that

$$
\left\|\Delta u_{n+1}\right\|_{\alpha} \leq \hat{\lambda}^{n}\left\|\Delta u_{1}\right\|_{\alpha}+\varepsilon \sum_{j=0}^{n-1} \hat{\lambda}^{j}=\hat{\lambda}^{n}\left\|\Delta u_{1}\right\|_{\alpha}+\frac{\varepsilon\left(1-\hat{\lambda}^{n}\right)}{1-\hat{\lambda}} .
$$

By selecting $\alpha$ such that $\hat{\lambda}<1$, then we have

$$
0 \leq \lim _{n \rightarrow \infty}\left\|\Delta u_{n}\right\|_{\alpha} \leq \frac{\varepsilon}{1-\widehat{\lambda}} .
$$

When $b_{\psi}=0$ and $b_{\eta}=0$, then $\varepsilon=0$. It is easy to obtain

$$
0 \leq \lim _{n \rightarrow \infty}\left\|\Delta u_{n}\right\|_{\alpha} \leq 0,
$$

which implies $\lim _{n \rightarrow \infty}\left\|\Delta u_{n}\right\|_{\alpha}=0$. 

has

Similarly, multiplying $(1 / \alpha)^{k}$ for both sides of (19), one

$$
\begin{aligned}
\left\|\Delta \mathbf{x}_{n}(k)\right\|\left(\frac{1}{\alpha}\right)^{k} & \\
& \leq \frac{1}{\alpha} \sum_{j=0}^{k-1}\left(\frac{M}{\alpha}\right)^{k-1-j}\left[b_{\mathbf{b}}\left\|\Delta u_{n}(j)\right\|\left(\frac{1}{\alpha}\right)^{j}+\left(\frac{1}{\alpha}\right)^{j} b_{\psi}\right] .
\end{aligned}
$$

Because $\alpha>\max \left\{1, M, M+b_{\mathbf{b}} b_{\Gamma_{2}}\right\}$, then $(M / \alpha)<1$. Hence, (32) implies that

$$
\begin{aligned}
\left\|\Delta \mathbf{x}_{n}\right\|_{\alpha} & \leq\left(b_{\mathbf{b}}\left\|\Delta u_{n}\right\|_{\alpha}+b_{\psi}\right) \frac{1-(M / \alpha)^{K}}{\alpha-M} \\
& =b_{\mathbf{b}} \frac{1-(M / \alpha)^{K}}{\alpha-M}\left\|\Delta u_{n}\right\|_{\alpha}+b_{\psi} \frac{1-(M / \alpha)^{K}}{\alpha-M} .
\end{aligned}
$$

Since $b_{\mathbf{C}}=\|\mathbf{C}\|=1$, multiplying $(1 / \alpha)^{k}$ for both sides of (20), we can derive that

$$
\begin{aligned}
\left\|\mathbf{e}_{n}\right\|_{\alpha} & \leq\|\mathbf{C}\|\left\|\Delta \mathbf{x}_{n}\right\|_{\alpha}+b_{\boldsymbol{\eta}} \\
& \leq b_{\mathbf{b}} \frac{1-(M / \alpha)^{K}}{\alpha-M}\left\|\Delta u_{n}\right\|_{\alpha}+b_{\psi} \frac{1-(M / \alpha)^{K}}{\alpha-M}+b_{\eta} .
\end{aligned}
$$

Substituting (30) into (34) and taking the limit of $\left\|\mathbf{e}_{n}\right\|_{\alpha}$ yield

$$
0 \leq \lim _{n \rightarrow \infty}\left\|\mathbf{e}_{n}\right\|_{\alpha} \leq \frac{\varepsilon}{1-\widehat{\lambda}} b_{\mathbf{b}} \frac{1-(M / \alpha)^{K}}{\alpha-M}+b_{\psi} \frac{1-(M / \alpha)^{K}}{\alpha-M}+b_{\eta},
$$

when $b_{\psi}=0, b_{\eta}=0$, and $\varepsilon=0$. Hence, (35) implies

$$
0 \leq \lim _{n \rightarrow \infty}\left\|\mathbf{e}_{n}\right\|_{\alpha} \leq 0
$$

that is, $\lim _{n \rightarrow \infty}\left\|\mathbf{e}_{n}\right\|_{\alpha}=\lim _{n \rightarrow \infty}\left\|\mathbf{y}_{n}(k)-\mathbf{y}_{d}(k)\right\|_{\alpha}=0$.

Remark 11. It is noticeable from the analysis that the convergence of the learning system can be guaranteed under some sufficient conditions, which are independent of the stabilizing controller. This means that the feedforward learning mechanism can still work in the uncertain environment without the stabilizing controller, but the learning rate can be improved if a suitable one is used.

\section{Simulation Study}

Consider a long segment of freeway with the length of $3 \mathrm{~km}$, and there is only one on-ramp located in the segment. The maximum traffic density $\rho_{\max }=80 \mathrm{veh} / \mathrm{lane} / \mathrm{km}$. The traffic flow entering the mainline $f(k)$ and the inflow entering the ramp $r(k)$ are given in Figure 2, respectively.

Consider an on-ramp with about $100 \mathrm{~m}$ length; the desired queue length is $l_{d}=7 \mathrm{veh}$. The free speed $v_{f}=$ $120 \mathrm{~km}$ /hour, the sampling time $T=30 \mathrm{~s}$, and the finite sampling time instants $K=60$.

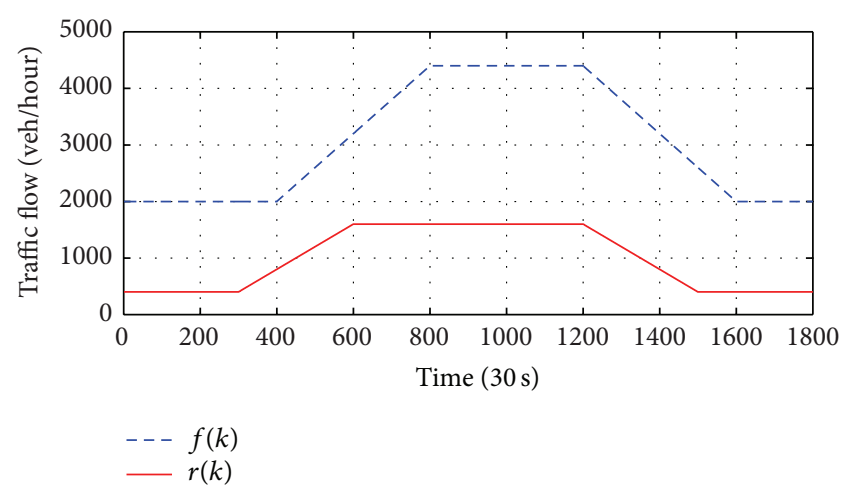

Figure 2: The traffic demands of the mainline and the on-ramp.

In the simulation, considering the inevitable initial resetting error, we assume that both the initial traffic density, $\rho_{n}^{i}(0)=40+\operatorname{rand}(\mathrm{veh} / \mathrm{lane} / \mathrm{km})$, and the queue length, $l_{n}(0)=7+$ rand $n$ veh, are nonstrictly repeatable. In addition, to show the robustness of the proposed approach, we set

$$
\begin{aligned}
& \psi_{n}(k)=\left[8 \sin \left(\frac{0.02 k}{3 n}\right) 5 \sin \left(\frac{0.01 k}{2 n}\right)\right]^{T}, \\
& \boldsymbol{\eta}_{n}(k)=\left[5 \sin \left(\frac{0.03 k}{5 n}\right) 2 \sin \left(\frac{0.02 k}{3 n}\right)\right]^{T} .
\end{aligned}
$$

Applying the proposed QLIF-ILC approach (12) with $\Gamma_{1}=$ $[1.53,0.27]$ and $\Gamma_{2}=[18.7,3.3]$, the simulation results are shown as the red solid lines in Figures 3 and 4, respectively. Figure 3 shows the learning convergence of traffic density with respect to the iteration number $n$. The horizon is the iteration number and the vertical axis is the maximum absolute values of the traffic density tracking error $e_{\max , n}^{1}=$ $\max _{k \in\{0, \ldots, 60\}}\left|\rho_{d}-\rho_{n}(k)\right|$. Figure 4 shows the learning convergence of queue length with iterations, where the vertical axis is the maximum absolute values of the queue length tracking error $e_{\max , n}^{2}=\max _{k \in\{0, \ldots, 60\}}\left|l_{d}-l_{n}(k)\right|$.

From Figures 3 and 4 , the asymptotic learning convergence along iteration axis of traffic density and queue length can be clearly seen, and the fluctuation in mainline flow and queue length is considerably reduced with iterations. Further, the proposed approach is robust for the uncertain disturbances that existed in system states, outputs, and initial conditions.

For the purpose of comparison, we design a PD-type ILC law similar to (12) according to [9] as follows:

$$
u_{n+1}(k)=u_{n}(k)+\beta_{1} \cdot\left[\delta_{n}(k+1)-\delta_{n}(k)\right]+\beta_{2} \cdot \delta_{n+1}(k),
$$

where $\delta_{n+1}(k)=\rho_{d}(k)-\rho_{n+1}(k)$ is the traffic density error only and $\beta_{1}$ and $\beta_{2}$ are constants denoting the learning gains. The only difference between (12) and (38) is that the latter does not contain information of the queue length directly in the control law and the queue length is used as the constraints exposed on the control input signals.

Applying the ILC scheme (38) with $\beta_{1}=1.53$ and $\beta_{2}=$ 18.7, the simulation results are shown as the blue dash lines in 
TABLE 1: The mean speed with iterations ( $\mathrm{km} /$ hour).

\begin{tabular}{lccccccc}
\hline Iteration number & 1 & 10 & 20 & 30 & 40 & 50 & 50 \\
\hline QLIF-ILC & 41.07 & 49.65 & 51.65 & 52.61 & 53.23 & 53.58 & 53.88 \\
Standard-ILC & 41.07 & 45.42 & 48.60 & 50.58 & 51.65 & 52.38 & 52.88 \\
Improvements & $0.00 \%$ & $9.32 \%$ & $6.28 \%$ & $4.01 \%$ & $3.06 \%$ & $2.29 \%$ & $1.89 \%$ \\
\hline
\end{tabular}

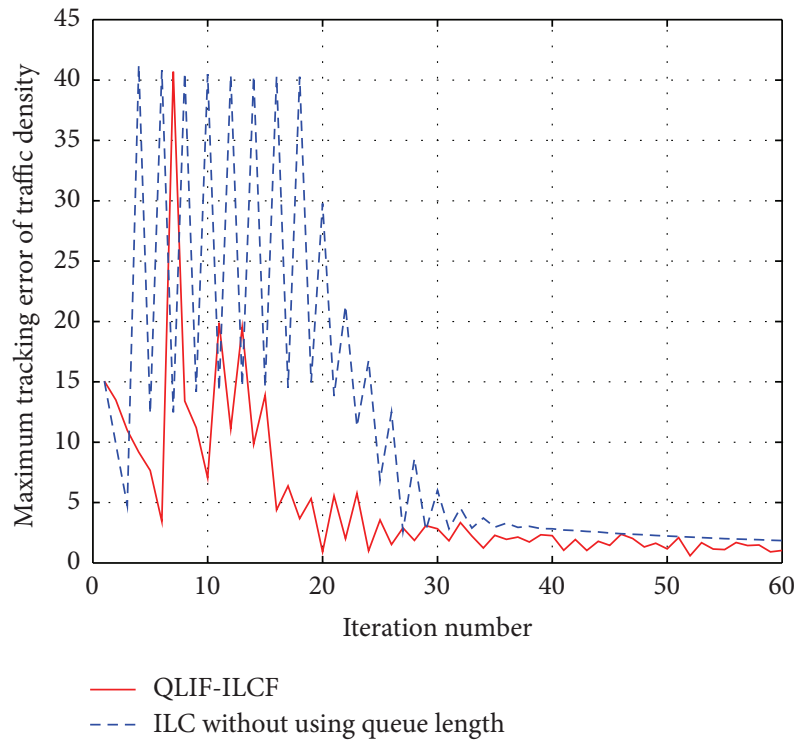

Figure 3: Maximum tracking error of macroscopic traffic flow density.

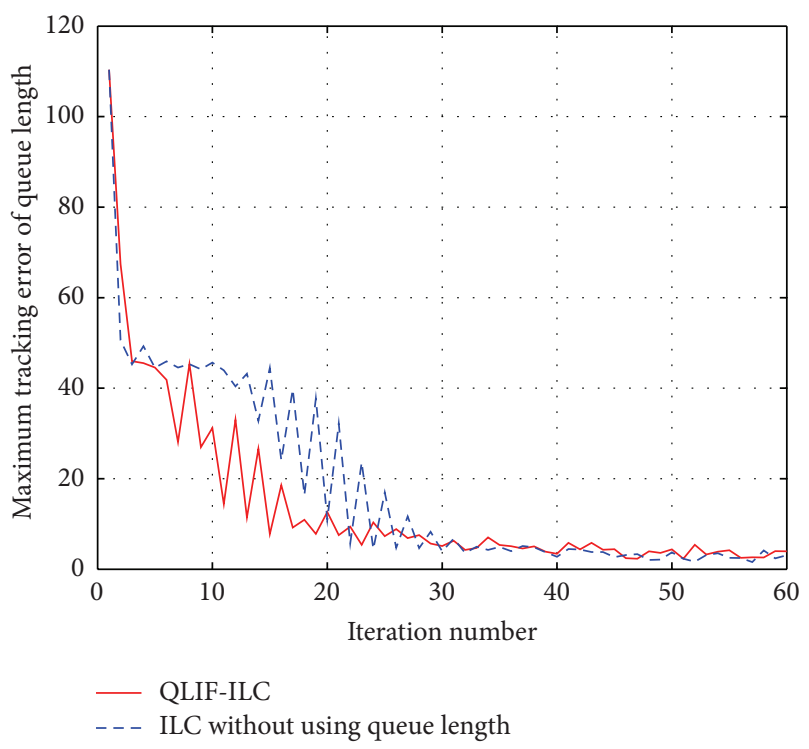

Figure 4: Maximum tracking error of ramp queue length.

Figures 3 and 4, respectively. It is noticeable that the proposed QLIF-ILC approach has a better performance than the ILC scheme (38) without using queue length information.

To further assess the control performance of the proposed QLIF-ILC approach (12) and the ILC without explicitly using queue length information (38), define a performance index of mean speed MS (km/hour) as

$$
\mathrm{MS}=\frac{\mathrm{TTD}}{\mathrm{TTS}},
$$

where TTS $=\sum_{n} \sum_{k} \sum_{i} m \cdot T \cdot \rho_{n}^{i}(k) \cdot L_{i}+\sum_{n} \sum_{k} T \cdot l_{n}(k)$ denotes the total travel time (veh.hour) and TTD $=\sum_{n} \sum_{k} \sum_{i} m \cdot T$. $\rho_{n}^{i}(k) \cdot v_{f}\left(1-\left(\rho_{n}^{i}(k) / \rho_{\max }\right)\right) \cdot L_{i}$ denotes the total travel distance (veh.km). Apparently, a larger mean speed means a better working of the traffic system.

When applying the proposed QLIF-ILC law (12) and the ILC law (38), respectively, the mean speed values are presented in Table 1. It can be seen that the proposed QLIFILC achieves an improved performance over the traditional iterative learning ramp metering schemes [9-11].

\section{Conclusion}

In this paper, a discrete iterative learning control based on the queue length information fusion (QLIF-ILC) is proposed for the nonlinear freeway traffic systems with input disturbance and output measurement noise, where a feedforward learning part is combined with a feedback stabilizing part. The onramp queue length is explicitly fused into the designed ILC mechanism to achieve a better performance. A systematic approach is developed to analyze the convergence and robustness of the proposed learning scheme. It is shown that the uniform boundedness between the system output and the desired output is achieved in each iteration. When all the uncertainties tend to zero, the system output will converge uniformly to the desired one. The simulation study on a macroscopic level freeway model confirms the validity of the proposed QLIF-ILC.

\section{Conflict of Interests}

The authors declare that they have no conflict of interests regarding the publication of this paper.

\section{Acknowledgment}

This work is supported by the National Natural Science Foundation of China (61120106009, 61374102, and 60974040).

\section{References}

[1] E. Smaragdis, M. Papageorgiou, and E. Kosmatopoulos, "A flowmaximizing adaptive local ramp metering strategy," Transportation Research B: Methodological, vol. 38, no. 3, pp. 251-270, 2004.

[2] T.-H. Chang and Z.-Y. Li, "Optimization of mainline traffic via an adaptive co-ordinated ramp-metering control model with 
dynamic OD estimation," Transportation Research C: Emerging Technologies, vol. 10, no. 2, pp. 99-120, 2002.

[3] X. Zhao, D. Srinivasan, and J. Xu, "Iterative learning control of freeway flow via ramp metering and simulation on PARAMICS," in Proceedings of the IEEE Region 10 Conference (TENCON '09), pp. 1-6, Singapore, November 2009.

[4] M. Papageorgiou and A. Kotsialos, "Freeway ramp metering: an overview," IEEE Transactions on Intelligent Transportation Systems, vol. 3, no. 4, pp. 271-281, 2002.

[5] A. Kotsialos, M. Papageorgiou, M. Mangeas, and H. Haj-Salem, "Coordinated and integrated control of motorway networks via non-linear optimal control," Transportation Research C: Emerging Technologies, vol. 10, no. 1, pp. 65-84, 2002.

[6] H. M. Zhang, S. G. Ritchie, and R. Jayakrishnan, "Coordinated traffic-responsive ramp control via nonlinear state feedback," Transportation Research C: Emerging Technologies, vol. 9, no. 5, pp. 337-352, 2001.

[7] H. B. Ji, "Freeway traffic systems: prediction and control," in Proceedings of the IEEE 46th Vehicular Technology Conference on "Mobile Technology for the Human Race", vol. 3, pp. 1815-1819, Atlanta, Ga, USA, 1996.

[8] F.-S. Ho and P. Ioannou, "Traffic flow modeling and control using artificial neural networks," IEEE Control Systems Magazine, vol. 16, no. 5, pp. 16-26, 1996.

[9] Z. Hou, J.-X. Xu, and H. Zhong, "Freeway traffic control using iterative learning control-based ramp metering and speed signaling," IEEE Transactions on Vehicular Technology, vol. 56, no. 2, pp. 466-477, 2007.

[10] Z. Hou, J.-X. Xu, and J. Yan, "An iterative learning approach for density control of freeway traffic flow via ramp metering," Transportation Research C: Emerging Technologies, vol. 16, no. 1, pp. 71-97, 2008.

[11] Z. Hou, X. Xu, J. Yan, J.-X. Xu, and G. Xiong, "A complementary modularized ramp metering approach based on iterative learning control and ALINEA," IEEE Transactions on Intelligent Transportation Systems, vol. 12, no. 4, pp. 1305-1318, 2011.

[12] S. Arimoto, S. Kawamura, and F. Miyazaki, "Bettering operation of robots by learning," Journal of Robotic Systems, vol. 1, pp. 123$140,1984$.

[13] C.-J. Chien, "A discrete iterative learning control for a class of nonlinear time-varying systems," IEEE Transactions on Automatic Control, vol. 43, no. 5, pp. 748-752, 1998.

[14] M. Sun and D. Wang, "Initial shift issues on discrete-time iterative learning control with system relative degree," IEEE Transactions on Automatic Control, vol. 48, no. 1, pp. 144-148, 2003.

[15] H.-S. Ahn, Y. Q. Chen, and K. L. Moore, "Iterative learning control: brief survey and categorization," IEEE Transactions on Systems, Man and Cybernetics C: Applications and Reviews, vol. 37, no. 6, pp. 1099-1121, 2007.

[16] J.-X. Xu and Y. Tan, Linear and Nonlinear Iterative Learning Control, vol. 291 of Lecture Notes in Control and Information Sciences, Springer, Berlin, Germany, 2003.

[17] R. Chi, Z. Hou, and J. Xu, "Adaptive ILC for a class of discretetime systems with iteration-varying trajectory and random initial condition," Automatica, vol. 44, no. 8, pp. 2207-2213, 2008.

[18] X. Sun and R. Horowitz, "A localized switching ramp-metering controller with a queue length regulator for congested freeways," in Proceedings of the American Control Conference (ACC '05), pp. 2141-2146, Portland, Ore, USA, June 2005.
[19] R. L. Gordon, "Algorithm for controlling spillback from ramp meters," Transportation Research Record, no. 1554, pp. 162-171, 1996.

[20] E. Smaragdis and M. Papageorgiou, "Series of new local ramp metering strategies," Transportation Research Record, no. 1856, pp. 74-86, 2003.

[21] P. Kachroo and K. Ozbay, Feedback Ramp Metering in Intelligent Transportation Systems, Kluwer Academic/Plenum Publishers, New York, NY, USA, 2003.

[22] B. D. Greenshields, "A study of highway capacity," in Proceedings of Highway Research Record, vol. 14, pp. 448-477, Washington, Wash, USA, 1935. 


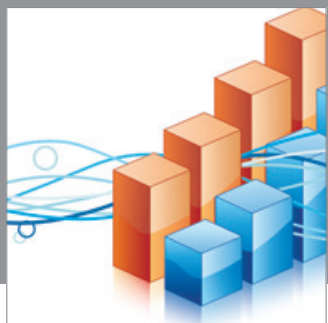

Advances in

Operations Research

mansans

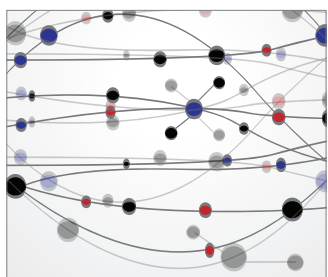

The Scientific World Journal
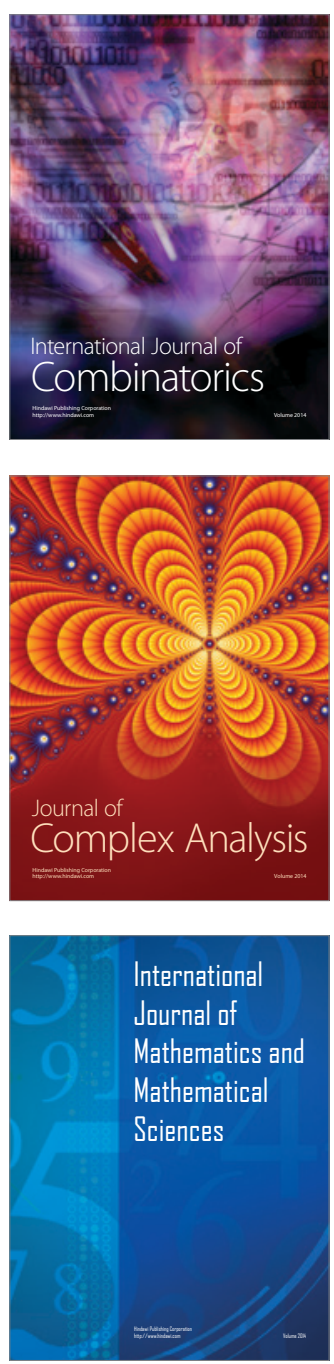
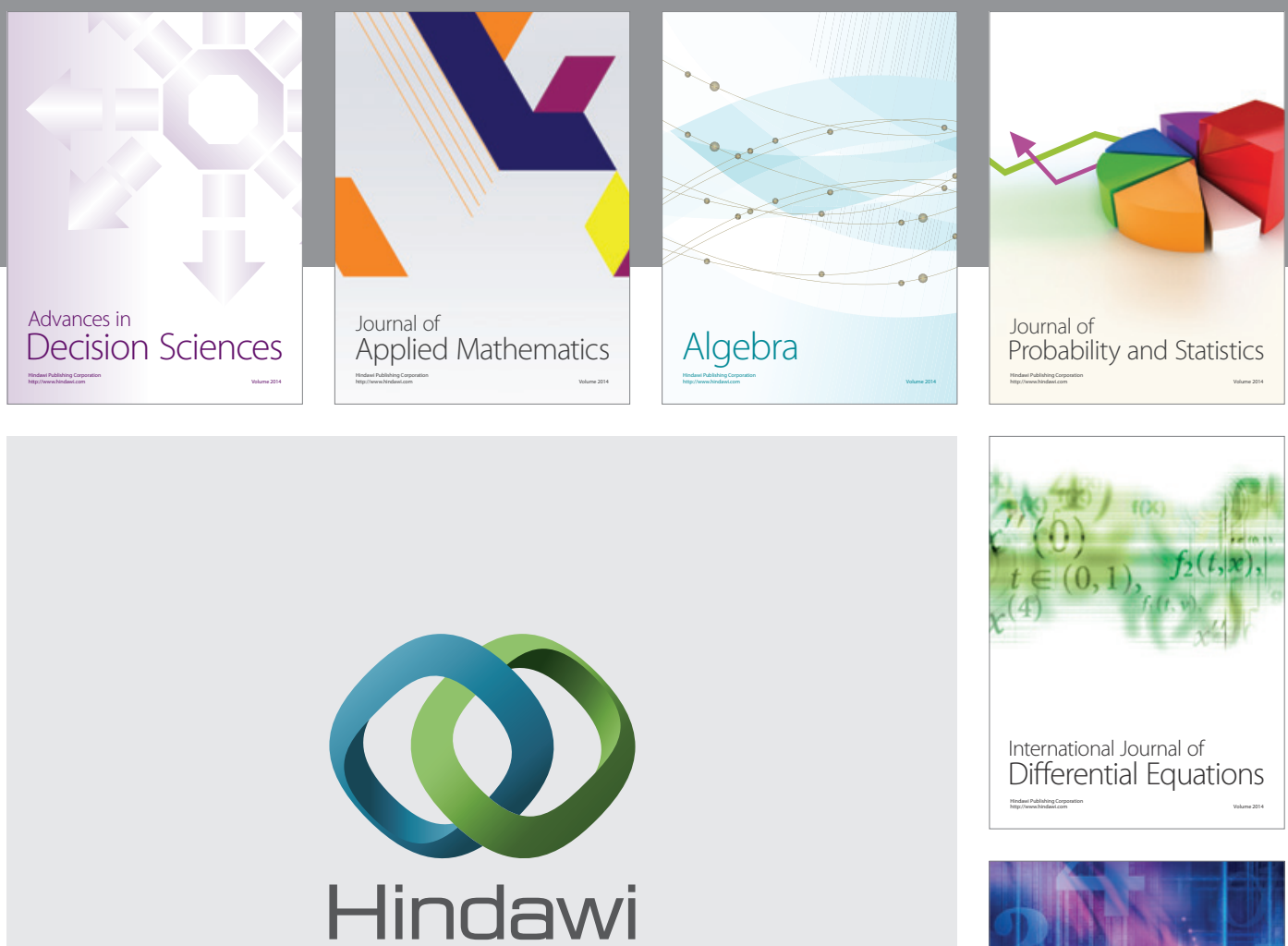

Submit your manuscripts at http://www.hindawi.com
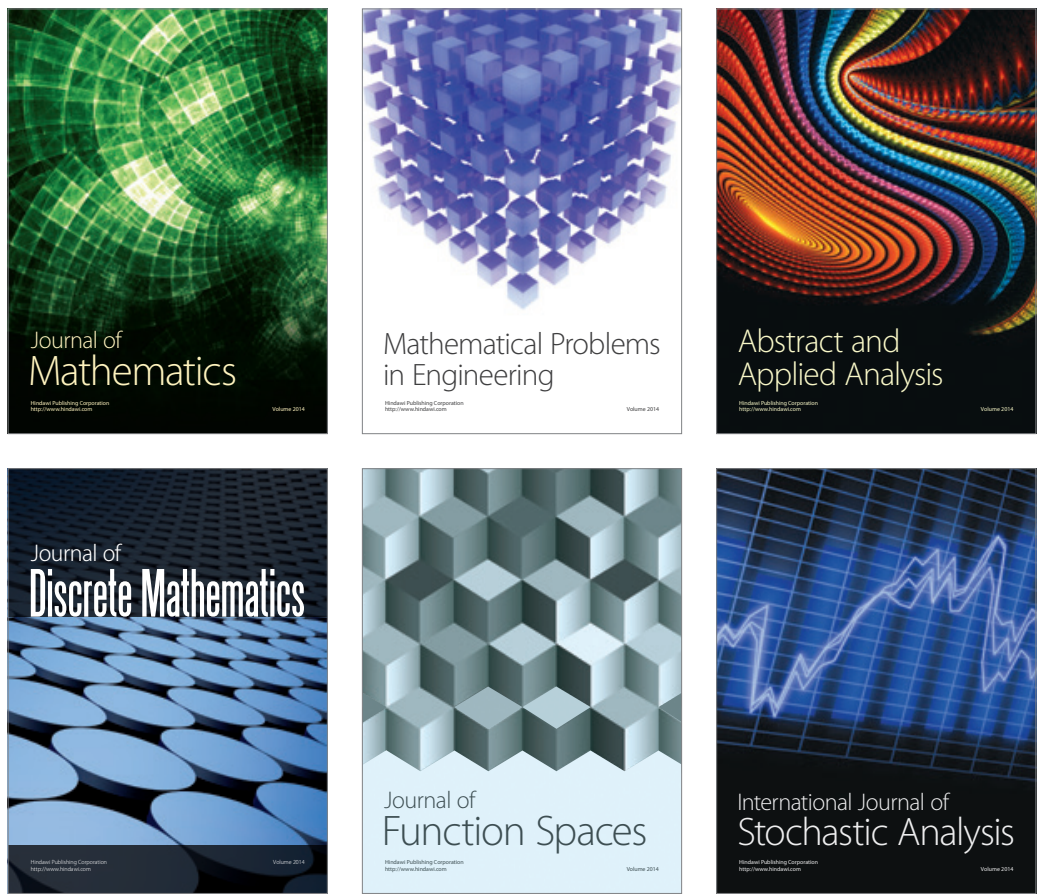

Journal of

Function Spaces

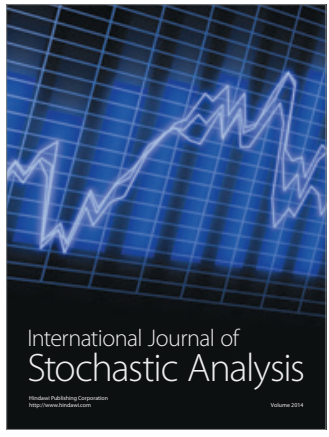

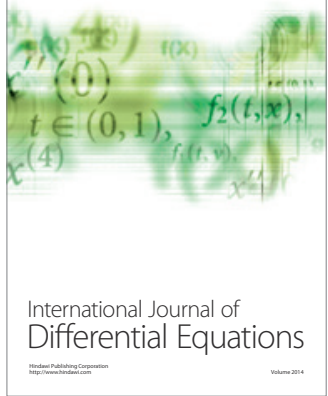
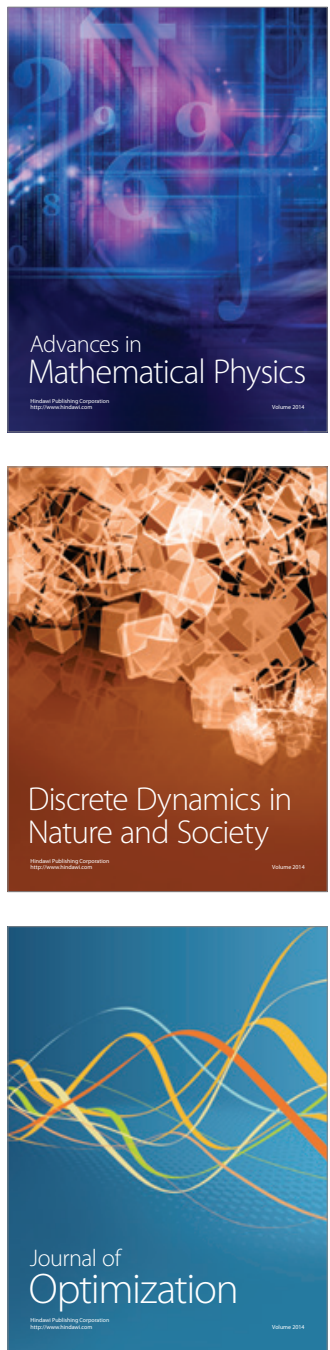